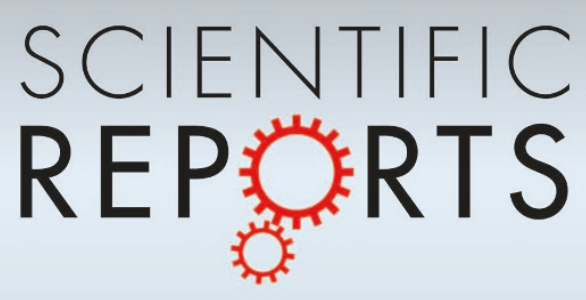

\title{
OPEN \\ The Open Gate Structure of the Membrane-Embedded KcsA Potassium \\ SUBJECT AREAS: \\ SINGLE-MOLECULE \\ BIOPHYSICS \\ Channel Viewed From the Cytoplasmic Side
}

SINGLE-CHANNEL RECORDING

COMPUTATIONAL BIOPHYSICS

ATOMIC FORCE MICROSCOPY

\author{
Ayumi Sumino ${ }^{1,2}$, Takashi Sumikama ${ }^{3}$, Masayuki Iwamoto ${ }^{3}$, Takehisa Dewa ${ }^{1,4} \&$ Shigetoshi Oiki ${ }^{3}$
}

Received

12 September 2012

Accepted

6 December 2012

Published

15 January 2013

Correspondence and requests for materials should be addressed to

T.D. (takedewa@ nitech.ac.jp) or S.O. (oiki-fki@umin.ac.jp)

\begin{abstract}
'Department of Frontier Materials, Graduate School of Engineering, Nagoya Institute of Technology, Nagoya 466-8555, Japan, ${ }^{2}$ Research Fellow of the Japan Society for the Promotion of Science, ${ }^{3}$ Department of Molecular Physiology and Biophysics, Faculty of Medical Sciences, University of Fukui, Fukui 910-1 193, Japan, ${ }^{4}$ PRESTO, Japan Science and Technology Agency (JST), Saitama 332-0012, Japan.
\end{abstract}

Crystallographic studies of channel proteins have provided insight into the molecular mechanisms of ion channels, even though these structures are obtained in the absence of the membrane and some structural portions have remained unsolved. Here we report the gating structure of the membrane-embedded KcsA potassium channel using atomic force microscopy (AFM). The activation gate of the KcsA channel is located on the intracellular side, and the cytoplasmic domain was truncated to clear the view of this location. Once opened, the individual subunits in the tetramer were resolved with the pore open at the center. Furthermore, AFM was able to capture the previously unsolved bulge helix at the entrance. A molecular dynamics simulation revealed that the bulge helices fluctuated dramatically at the open entryway. This dynamic behavior was observed as vigorous open-channel noise in the single-channel current recordings. The role of the bulge helices in the open gate structure is discussed.

$\mathrm{T}$ he potassium channel exists ubiquitously on the cell membrane, and maintains cellular electrical activity ${ }^{1}$. A vast array of gene species for the potassium channel has been identified across the whole range of living organisms $s^{2}$. The crystal structures of several channel types have been solved and the basic architecture was elucidated $^{3-7}$. The channel structure is functionally divided into the pore domain and the sensory domain. The pore domain is responsible for the common channel function, i.e. the selective $\mathrm{K}^{+}$permeation and gating. In contrast, the sensory domain, which is located in the cytoplasm or membrane, modulates the gating function of the pore domain. In this arrangement, the environmental changes sensed by the various types of the sensory domain are transferred to the pore domain so as to initiate channel activity. Thus, the pore domain is the canonical structural unit for all of the potassium channels ${ }^{8}$, and elucidating the gating conformation of the pore domain is thus crucial for an understanding the channel function.

The high resolution structure of the pore domain was first revealed for the KcsA potassium channel (Fig. 1) ${ }^{9}$, and the ion permeation mechanism through the selectivity filter has been reported ${ }^{10-14}$. The open conformation of the KcsA channel ${ }^{15}$ as well as that of several potassium channel types ${ }^{16,17}$ demonstrated that the crossed bundle helices (the M2 helix) at the intracellular end of the pore domain relax, which then allows ions to have access to the pore. The full-length structure of the KcsA channel was subsequently resolved ${ }^{18,19}$, showing the straight helix bundle to be the cytoplasmic domain (CPD). However, these structural data were obtained in the absence of the membrane, and several maneuvers, including the introduction of mutations and fixation with antibodies should have been taken for crystallization, which would bias the intact structure.

Here we report the structure of the activation gate in the pore domain of the KcsA channel in the membraneembedded state, and atomic force microscopy (AFM) is shown to be useful for capturing such global conformational changes upon gating in the native state. The AFM provides the surface contour, and thus the structural parts protruding from the membrane surface can be delineated ${ }^{20-23}$. For certain membrane proteins, a bulky oligomeric domain, protruding into either the extracellular or intracellular space, has been resolved by $\mathrm{AFM}^{24-26}$, and the conformational change of the extra-membranous parts is relevant to the sensory process. In contrast, our focus is the less bulky, membrane-embedded pore domain with the activation gate open and closed. In the various 
Extracellular
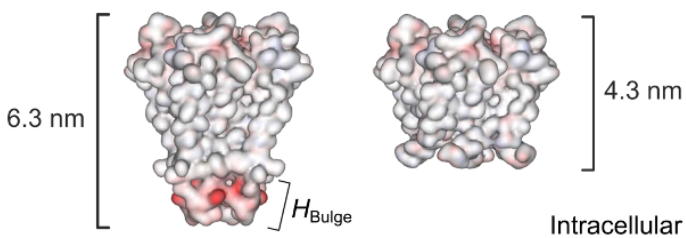

Intracellular

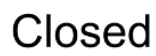

Figure $1 \mid$ Crystal structure of the closed (residue 22-124) and open (residue 30-117) conformations of the KcsA channel (pdb code: $1 \mathrm{~K} 4 \mathrm{C}$ and 3F5W). The longitudinal length of the channel was calculated from the crystal structure as the distance between the $\mathrm{C}_{\alpha}$ atoms furthest apart along the channel axis plus their atomic size. For the closed conformation the unresolved terminal residue (F125) is taken into considerations. The surface of the channel is colored for the charge distribution (red for negative and blue for positive).

potassium channels the pore domain shares a common architecture, and some degree of homologous gating transition should take place. What configuration does the activation gate take? How do ions in the bulk intracellular solution gain access to the pore through the open gate?

In the pore domain, including that in the KcsA channel, the activation gate is located close to the cytoplasmic end, where the global conformational changes manifest ${ }^{27,28}$. To observe the open and closed gate of the membrane embedded KcsA potassium channel from the cytoplasmic side, the CPD, which is formed by a straight helix bundle and hence hinders the view, was truncated. The CPDtruncated KcsA channel retains its native functions of $\mathrm{pH}$-dependent gating and single-channel activity ${ }^{29}$. Between the pore domain and the CPD, there is a structural region known as the bulge helix (residue 118-135; Fig. 1) ${ }^{18}$. The charged residues are clustered there and function as a part of the intracellular $\mathrm{pH}$-sensor ${ }^{29-31}$. The bulge helix is a critical structure that governs the size for the ion accessing aperture in the open conformation. In the CPD-truncated channel, one half of the bulge helix is retained at the C-terminal end (118-125), and we call this region the part of the bulge helix $\left(H_{\text {bulge }}\right)$. In the crystal structure of the open state, the $H_{\text {bulge }}$ has remained unsolved.

In this study, the CPD-truncated KcsA channel embedded in the membrane was the target of structural and functional investigation in the single-molecule level. In the AFM image that was obtained, the tetrameric open conformation was revealed, and the crystallographycally-unsolved $H_{\text {bulge }}$ was captured. The structure of the $H_{\text {bulge }}$ was generated by means of a simulation with molecular dynamics (MD), by which the dynamic behavior was examined. Finally, the functional consequences of the fluctuating structure were studied using singlechannel current recordings. This integrated single-molecule approach using AFM, MD simulation and single-channel current recordings afforded a clear picture of the dynamic structure and function of the activation gate of the KcsA channel.

\section{Results}

Images of the KcsA in the membrane. The liposomes reconstituted with the CPD-truncated KcsA channel (residue 1-125) were extended over the mica surface, and AFM measurements were performed. Distinct islands of flat membranes became evident and protruding particles were distributed exclusively on the membrane surface (Fig. 2). The individual particles observed at pH 7.5 (Fig. 2A) and 4.0 (Fig. 2B) were found to be substantially different in size. These particles represent the cytoplasmic end of the CPD-truncated KcsA channel for the following reasons. In the reconstituted liposomes, the KcsA channel is oriented in the "right-side out" configuration (i.e. the cytoplasmic domain faces to the inside vesicle). This orientation has been confirmed by functional measurements using liposome-patch and the planar lipid bilayer methods exploiting the location of the $\mathrm{pH}$-dependent sensor of the KcsA channel on the cytoplasmic side ${ }^{30,32}$. Thus, the KcsA channel was in an upside-down orientation (i.e., the cytoplasmic side up) in the flat bilayer.

KcsA shortens its longitudinal length upon opening. The height profile indicates a flat bilayer membrane with a thickness of approximately $4 \mathrm{~nm}$ on which surface protrusions are present (Fig. 2A, B, lower panels). The histograms show that the height from the mica surface, or the longitudinal length of the channel, were significantly different at the two different $\mathrm{pH}$ values (Fig. 2C): $6.4 \pm 0.3 \mathrm{~nm}$ at pH $7.5(\mathrm{~A})$ and $4.9 \pm 0.4 \mathrm{~nm}$ at $\mathrm{pH} 4.0$ (B). A substantial shortening of the open pore was clearly resolved by AFM.

While this shortening was expected from the crystal structures of the pore domain in the closed and open conformations ${ }^{9,15}$ (Fig. 1), the degree of the shortening was not, since the structure of several of the residues in the cytoplasmic end in the open conformation has remained unsolved. Thus, the AFM image was compared to the crystal structure in terms of the longitudinal length of the channel. The height for the closed conformation obtained from AFM (6.4 \pm $0.3 \mathrm{~nm})$ matches well with that of the crystal structure $(6.3 \mathrm{~nm}$; blue arrowhead). In the case of the open conformation, there is a substantial discrepancy between the height of the AFM image $(4.9 \mathrm{~nm})$ and that of the crystal structure $(4.3 \mathrm{~nm} \text {; red arrowhead })^{9}$. This discrepancy is schematically shown as the light blue square (Fig. 2D), and AFM captured the structure of the crystallographically-unsolved Cterminal end in the open conformation.

The open gate and the tetrameric structure. The channels were observed at a higher magnification (Fig. 3). The individual particles are well resolved for the open channel (Fig. 3B), having a half-width of $6.0 \mathrm{~nm}$ in the height profile. For the closed channel, on the other hand, large particles are seen (Fig. 3A), and the coarsegrained particles within them are resolved. The height profile shows a periodicity with a half-width of $6.0 \mathrm{~nm}$. This size matches the open channel, suggesting that the channel is clustered at a neutral $\mathrm{pH}$.

Further magnification images of the open gate at an acidic $\mathrm{pH}$ (4.0) are shown (Fig. 3C). The individual subunits in the tetrameric structure along with the hole in the center are clearly resolved. In contrast, the tetrameric structure was not resolved for the closed conformation (Fig. 3A inset). The six images are snapshots of the open gate (Fig. 3C), which would evoke the dynamic picture of the gating. In the height profile, a shallow dent is observed in the middle of the protrusion (Fig. 3B, the arrow in the lower panel), representing the open entrance. These images comprise the first observation of the open gate structure of the membrane-embedded KcsA channel. Averaging the open gate images reveals the distinct subunit assembly around the pore (Fig. 3D). From the height profile of the open conformation, the distance between the subunits was measured for the peaks in the subunits. The distance between the diagonal subunits was $4.4 \pm 0.3 \mathrm{~nm}(\mathrm{n}=10)$ and that between the adjacent subunits was $3.3 \pm 0.3 \mathrm{~nm}(\mathrm{n}=11)$. These values are clearly not in agreement with those of the crystal structure $(3.9 \mathrm{~nm}$ and $2.8 \mathrm{~nm}$ ).

The evident discrepancies in the molecular height and aperture size between the AFM images and the crystal structure indicate that the crystallographycally unsolved C-terminal residues, involving the $H_{\text {bulge, }}$ extend towards the cytoplasmic space. In general, vigorously fluctuating structural regions remain unsolved in crystallography. Therefore, to obtain a dynamic picture of the AFM-captured structure, the terminal regions were reproduced using a computer simulation method.

Fluctuation of the $\boldsymbol{H}_{\text {bulge }}$ detected by MD simulation. To generate the most plausible structure and configuration of the $H_{\text {bulge }}$ at the open pore entrance, the relevant regions were grafted to the open 

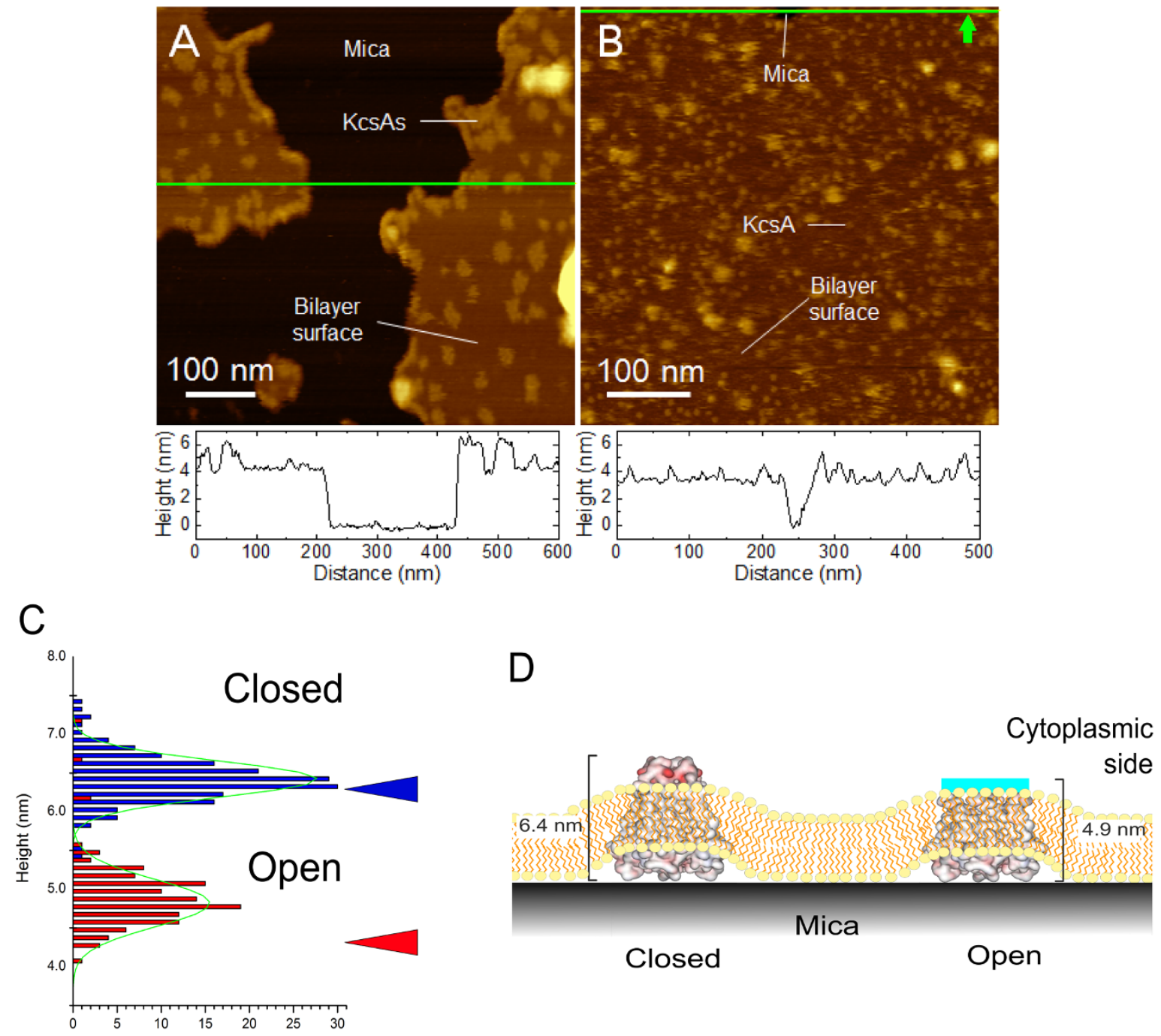

Figure $2 \mid$ KcsA images embedded in the membrane. (A, B) AFM images of the CPD-truncated KcsA channel in lipid bilayer and the height profiles. The KcsA-reconstituted proteoliposomes were added onto the mica surface, and the liposome membrane was extended on the mica substrate, forming the flat bilayer. The cytoplasmic part of the KcsA channel protruded from the membrane surface. The $\mathrm{pH}$ of the overlaying buffer solution was 7.5 for A and 4.0 for B. The height profiles along the green solid lines are shown below the images. Images were recorded with the acoustic AC mode of AFM. (C) Height distribution pattern of the CPD-truncated KcsA on the mica surface. The height was evaluated as the sum of the protruded height and the membrane thickness. The blue bars indicate the histogram for the closed conformation at $\mathrm{pH} 7.5$ and the red ones the open conformation at $\mathrm{pH} 4.0$. The green lines indicate the results of Gaussian fitting. The height was $6.4 \pm 0.3 \mathrm{~nm}$ at $\mathrm{pH} 7.5(\mathrm{n}=170)$ and $4.9 \pm 0.4 \mathrm{~nm}$ at $\mathrm{pH} 4.0(\mathrm{n}=121)$. The blue and red arrows indicate the height values obtained from the crystallographic structures for the closed $(6.3 \mathrm{~nm})$ and open $(4.3 \mathrm{~nm})$ states. (D) Schematic representation of the membrane-embedded channel on the mica surface. The intracellular structure protruding from the membrane surface was measured with AFM. The light blue box shown on the cytoplasmic end of the open conformation represents an AFM-retrieved structural part.

crystal structures, and an MD simulation of the CPD-truncated transmembrane domain (residues 22-125) in the membraneembedded condition was performed.

The structural configuration of the $H_{\text {bulge }}$ in the fully-open structure was obtained using a novel method exploiting structural templates with various degrees of pore opening. In the closed conformation, the crystallographic data resolved nearly all of the aminoacid residues of the CPD-truncated channel up to residue 124 . On the other hand, the available open structures with various degrees of opening left the $H_{\text {bulge }}$ unsolved ${ }^{15}$. Starting from the closed structure (pdb code: $1 \mathrm{~K} 4 \mathrm{C}$ ), the transmembrane region was made to move gradually to the templates of successively more widely open conformations (pdb code: 3F7Y, 3F7V, 3F5W; see the Methods section). The partially neutrarized $H_{\text {bulge }}$ at acidic $\mathrm{pH}$ (see Methods) was grafted at the end of the templates, and the connecting portion between the $H_{\text {bulge }}$ and the transmembrane region was moved farther apart in an centrifugal manner. Meanwhile the $H_{\text {bulge }}$ protruding towards the cytoplasmic space was allowed to move freely.

Typical structures in the closed and open states obtained by the MD simulation are shown in Fig. 4. The average longitudinal length of the simulated closed structure was $6.4 \pm 0.05 \mathrm{~nm}$, which is in good agreement with both the crystal structure and the AFM images (Fig. 4A). In contrast, the longitudinal length of the open structure was elongated substantially compared with that shown in both the AFM images and the crystal structure. The average length of the channel in the open state was estimated to be $5.4 \pm 0.13 \mathrm{~nm}$, which is longer than that in the AFM images by $0.5 \mathrm{~nm}$ (shown in magenta in Fig. 4C,D). Moreover, the average distance of the diagonal subunits is $4.5 \mathrm{~nm}$, which is slightly more separated compared with that in the AFM images (Fig. 4D), while no differences were seen in the closed conformation (Fig. 4B). These differences in the open structure between the AFM images and MD simulation indicate that the 

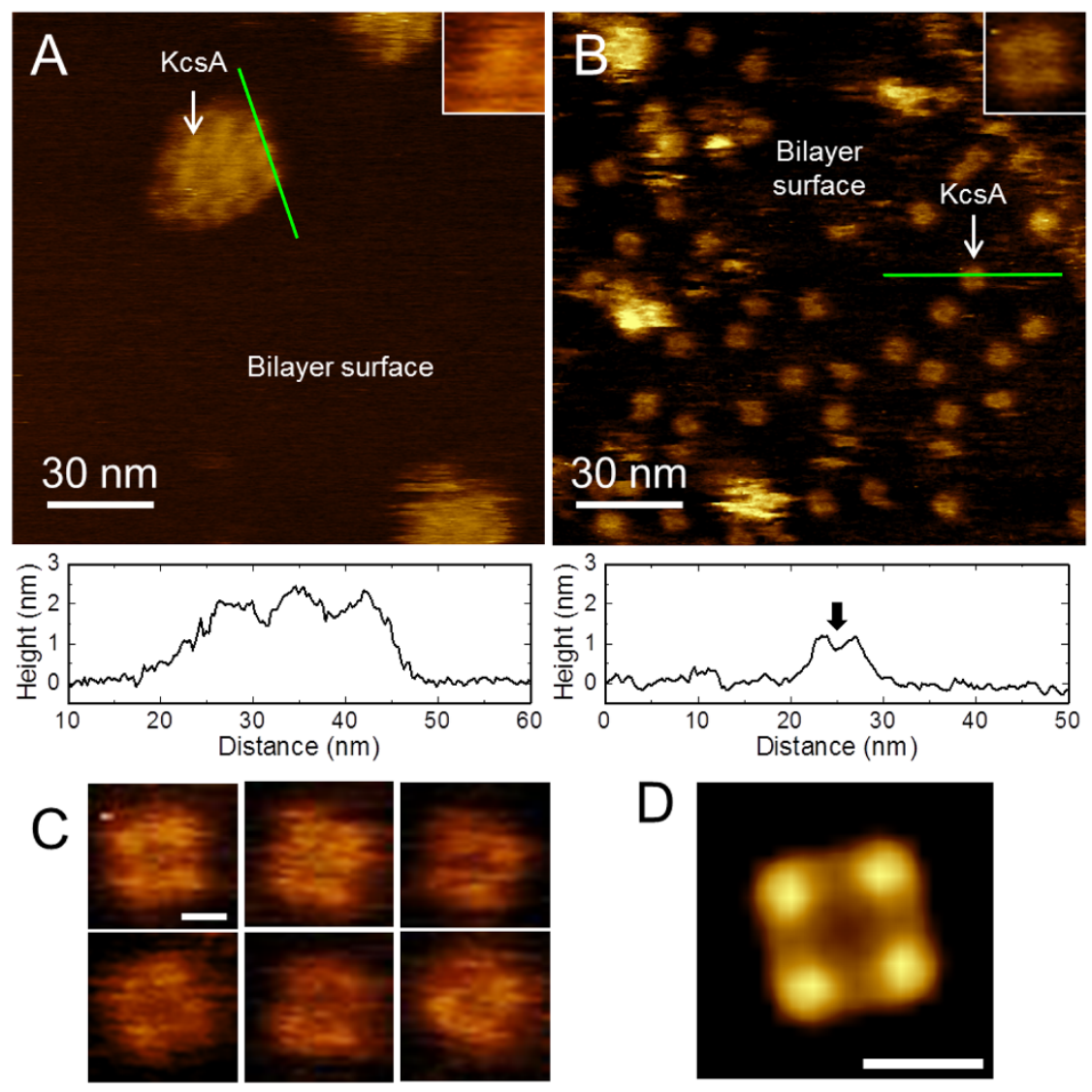

Figure 3 Structural images of the cytoplasmic end of the membrane-embedded KcsA channel. (A,B) Cytoplasmic views of the KcsA channel at pH 7.5 (A) and $\mathrm{pH} 4.0$ (B) and their height profiles along the green line. Particles are resolved in an island at neutral pH, while individual particles are identified at acidic pH. (Inset) A 3-times enlarged view of the arrowed KcsA channels. (C) A further magnified view of the open KcsA channel at acidic pH. The scale bars indicate $3 \mathrm{~nm}$. (D) An averaged AFM image of the open pore at acidic pH. The averaging was performed from 14 images of the open KcsA channels from Figure 3B. The scale bars indicate $3 \mathrm{~nm}$.

MD simulation complemented the very end of the $H_{\text {bulge }}$ in the open gate.

The MD simulation showed that the $H_{\text {bulge }}$ sticks out into the cytoplasmic space in the open state and fluctuates substantially (in yellow and magenta), while the main transmembrane regions (cyan) maintain an approximate 4 -fold symmetry (see the Supplemental video, Movie 1). This flexibility is represented as the B-factor, i.e. the temperature factor related to the structural fluctuation ${ }^{33}$ (Fig. 4E). In both the open and closed states, the B-factors of the transmembrane helices obtained by the MD simulation were coincident with that of the closed $(1 \mathrm{~K} 4 \mathrm{C})$ structure, but smaller than the open (3F5W) structure. In contrast, the B-factors of the portions sticking out into solution (the extracellular loop and both ends) exhibited a higher value due to thermal fluctuations. In particular, the extremely high B-factor of the $H_{\text {bulge }}$ clearly reflects its fluctuation. On the other hand, in the closed state, the cytoplasmic ends form a bundle crossing, so the fluctuations are suppressed (see the Supplemental video, Movie 2).

The four $H_{\text {bulge }}$ regions exhibited different structural configurations, and these were interconvertible with each other on a slower time scale (Movie 1). In fact, changes in the structural configurations of each of the helices were observed several times over the total simulation time of $200 \mathrm{~ns}$, and once a helix obstructed the pore, it did so for 5 to $10 \mathrm{~ns}$ or more. These results suggest that the movement of each helix has slower transition kinetics, and the MD simulation picked up some of these events.

The fluctuating $\boldsymbol{H}_{\text {bulge }}$ chops up the ionic current. To determine the relationship of the structural fluctuation of the $H_{\text {bulge }}$ observed in the
MD simulation to the functional behavior of the channel, singlechannel current measurements were performed. Here we examined a non-inactivating mutant of the KcsA channel, E71A, without a CPD, such that the functional features of the activation gate are demonstrated (Fig. 5) ${ }^{34}$. The single-channel currents of the E71A mutant channels at $\mathrm{pH} 4.0$ revealed that the activation gate is kept open more than $90 \%$ of the time, during which period vigorous fluctuations in the current are observed (Fig. 5A). Brief and frequent intermissions towards the non-conductive state present, and these fast events could not be fully resolved under the limited bandwidth of the current recordings (a $2 \mathrm{kHz}$ cut-off frequency). The amplitude histograms were drawn with the vertical axis in the logarithmic scale such that the current noise was clearly evident ${ }^{35,36}$. Compared to the Gaussian distribution (the solid curve) the histogram of the open state shows a one-sided shoulder with a long lasting tail, which is consistent with the presence of unresolved brief closures ${ }^{35,37}$. A sublevel (the red arrow head) is evident in both the current traces and the amplitude histogram.

To address the fluctuating current in relation to the fluctuating motion of the $H_{\text {bulge }}$, complete deletion of the $H_{\text {bulge }}$ might be helpful. However, the deletion would lead to a loss of $\mathrm{pH}$ sensitivity, and is thus inappropriate for functional measurements. Alternatively, the addition of the cytoplasmic domain imposes a load on the freely fluctuating bulge helices. Thus, the KcsA channel with and without the CPD makes it possible to compare the single channel behavior in order to elucidate the role of the CPD and the activity of the $H_{\text {bulge. In }}$ Fig 5B, the single-channel currents of the full-length channel are shown. With the CPD, the current fluctuation was attenuated 

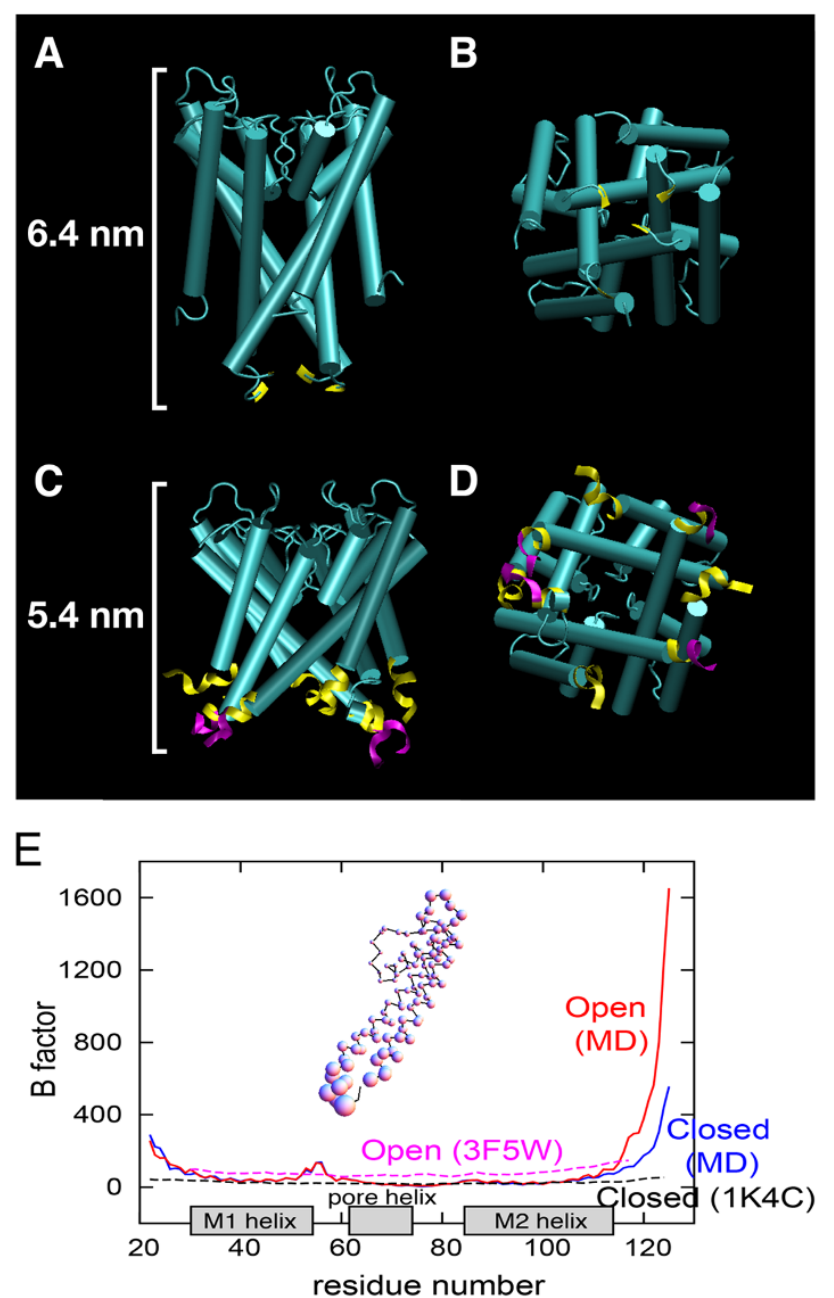

Figure $4 \mid$ Dynamics of the CPD-truncated transmembrane structure of the KcsA channel according to the MD simulation. (A) The side view of the closed structure (the cytoplasmic side up). (B) The bottom view of the closed structure. In the MD-simulated structure, the transmembrane region is colored cyan, which is similar to the X-ray crystallographic structure. The cytoplasmic end, that missed in the X-ray crystallographic structure is shown in yellow. (C) The side view of the open structure.

(D) The bottom view of the open structure. The transmembrane region is colored cyan, which is similar to the X-ray crystallographic structure. The cytoplasmic end, which is colored in yellow, constitutes the plausible structure that the AFM images detected. The structural parts, colored magenta, make up the terminal structure that the MD simulation resolved. The membrane was not shown in these figures. (E) The B-factor was calculated from the MD simulation. The red and blue lines represent the Bfactor obtained from the MD simulation for the open and closed structures, respectively. The B-factors of the crystal structures in open and closed states are also shown (the broken lines). The B-factors for residues 118-125 are significantly large. The B-factor for the extracellular loop was substantially higher than the intra-membrane region. (Inset) A subunit of the open configuration is shown, assigning the B-factors for the $\mathrm{C}_{\alpha}$ atoms with the volume of the spheres.

substantially, although the open probability was unchanged. The shoulder of the open channel in the histogram was depressed substantially.

The more frequent transition to the non-conducting state for the CPD-truncated channel indicates that the bulge helices freely fluctuate and transiently obstruct the conductive pathway. This choppy activity is attenuated but not eliminated in the presence of the CPD, which restricts the free movement of the bulge helices. The sub-level activity occurred exclusively for the CPD-truncated channel, and this suggests that the freely moving bulge helices incompletely closed the conductive pathway.

\section{Discussion}

In this study, a dynamic picture of the open gate for the KcsA channel embedded in the membrane was obtained by means of an integrated single-molecule approach. AFM revealed the novel findings that the cytoplasmic end of the transmembrane pore domain protruded from the membrane surface and the longitudinal length was substantially shortened upon opening. The high-resolution AFM images render the clear distinction between the closed and open gate stracture. In the open conformation, the tetrameric subunits were separately resolved with the pore open at the center. While the longitudinal length of the closed conformation was in agreement with that of the crystal structure, the substantially elongated and widened aperture relative to the crystal structure was also elucidated for the open conformation. The AFM-captured structural regions included the

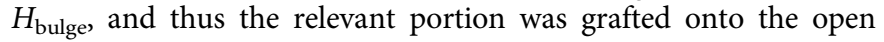
crystal structure in the course of the MD simulation. The MD simulation depicted the dynamics of the $H_{\text {bulge }}$ in the open gate, which manifested as open-channel noise in the single-channel current as the result of a "chopping up" of the entryway.

The time span of the $H_{\text {bulge }}$ fluctuation in the MD simulation ( $<$ hundreds of ns) and the single-channel current recordings ( $>$ hundreds of $\mu$ s) differs substantially. The end of the $H_{\text {bulge }}$ observed in the MD simulation moves rapidly, in the time range of ns, and the $H_{\text {bulge }}$ moves as a rigid-body, with a correspondingly slower time scale. The random hits for each helix and maintaining the obstructed configuration for a period of several ns led to the collective motions of the helices to slam the open pore shut. This stochastic event is likely to be both rare and brief, in a time range of $\mu$ s. On the other hand, the fluctuating single-channel current traces suggested events in the time range of sub-ms. However, based on the theoretical consideration of the open channel noise ${ }^{35}$, the asymmetric shoulder of the amplitude histogram confirms that the underlying events occur in a time span of $\mu$ s. Thus, a gap in the time span for the two results in the MD simulation and the single-channel current data is merging.

The open-channel noise in the single-channel current was vigorous in the CPD-truncated KcsA channel (Fig. 5). By restoring $\mathrm{CPD}$ as a full-length channel, the current fluctuation was substantially attenuated but still remained. Thus, CPD restricts but does not completely eliminate the flexible movement of the $H_{\text {bulge }}$. What is the functional role of the fluctuation that takes place in the bulge helices? The fluctuating behavior is likely an inherent property of the highly charged $\mathrm{pH}$ sensor ${ }^{29,31}$ which bears net positive charges at acidic $\mathrm{pH}$. Earlier reports demonstrated that the $\mathrm{pH}$-dependence was reduced by deleting the CPD (The Hill coefficient of the $\mathrm{pH}$ sensitivity was shown to be 0.7 for the CPDtruncated channel, rather than 1.4 for the full-length channel ${ }^{29}$ ). Based on these findings, we hypothesize that there is a functional role of the bulge helix. At neutral $\mathrm{pH}$, the bulge helices are stably bundled through either electrostatic interaction or hydrogen-bond formation. At acidic $\mathrm{pH}$, the net positive charges act as a repulsive force in the bulge helices ${ }^{29}$, leading to their fluctuation. In the absence of $\mathrm{CPD}$, vigorous fluctuations in each helix may drive the opening of the activation gate in each subunit. On the other hand, in the full-length channel with the CPD present, and hence restricting the free motion of the bulge helices, the individual movements of the bulge helices cooperate so as to induce a concerted effect on the activation gate that maximizes $\mathrm{pH}$-sensitivity.

Efforts are currently underway to gain insight into the continuous spectrum of the structural and functional dynamics of channel proteins. 

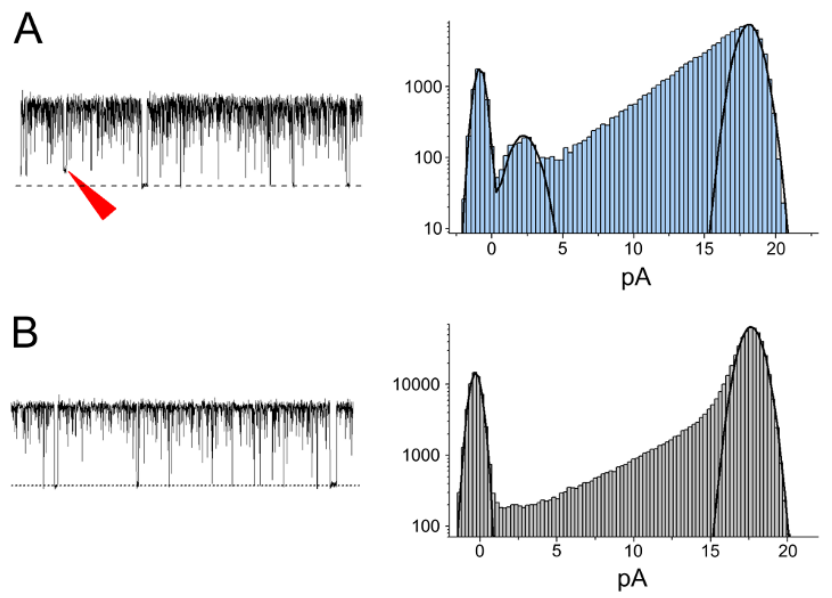

Figure $5 \mid$ Single-channel current at $+100 \mathrm{mV}$ without (A) and with (B) $\mathrm{CPD}$ at $\mathrm{pH}$ 4.0. The all-point amplitude histograms are shown with the vertical axis in the logarithmic scale, and the peaks are fitted with the Gaussian function. The shoulder of the open conductance peak is prominent for the CPD-truncated channel, indicating the open-channel noise from the highly fluctuating gating. A low conductance sub-level (the red arrowhead) was seen for the CPD-truncated channel.

\section{Methods}

Materials. The KcsA channel was purified as described previously ${ }^{38}$. Cytoplasmic domain (CPD)-truncated KcsA was prepared with previously reported procedure ${ }^{27}$. Phospholipid and detergent used in this study are L- $\alpha$-Phosphatidylcholine Type IV-S (asolectin; SIGMA) and n-Dodecyl- $\beta$-D-maltoside (DDM; Dojindo).

Reconstitution of KcsA into liposome. The phospholipid was dissolved in chloroform and then the solvent was evaporated under a stream of nitrogen. The resulting lipid film was dried in vacuo for at least $6 \mathrm{~h}$. The dry thin film was hydrated with a buffer solution (10 mM HEPES/NaOH (pH 7.5), $200 \mathrm{mM} \mathrm{KCl)}$ ), thereby providing a liposomal suspension. A DDM solution was added to the suspension to give a co-micellar solution ( $0.06 \mathrm{wt} \% \mathrm{DDM}$ of the final concentration). The solution of the KcsA dissolved in a buffer (0.06 w\% DDM in $10 \mathrm{mM} \mathrm{HEPES/NaOH} \mathrm{(pH} \mathrm{7.5),}$ $200 \mathrm{mM} \mathrm{KCl}$ ) was added to this co-micellar solution (in the range of 3/1-5/1 of lipid/ protein ratio $(w / w))$, and incubated for at least $1 \mathrm{~h}$ at $4^{\circ} \mathrm{C}$. After incubation, the co-micellar solution was dialyzed against detergent-free buffer for $30 \mathrm{~h}$ and stored at $4^{\circ} \mathrm{C}$. The proteoliposomal suspension was used for experiments within 1 week.

Liposome extension on the mica surface and AFM observation. Flat lipid bilayers on a mica surface were prepered with vesicle fusion method ${ }^{39,40}$. The proteoliposomal suspension was put onto a mica substrate after the addition of $\mathrm{MgCl}_{2}$ solution. After $\sim 15 \mathrm{~min}$ of incubation, the solution was gently replaced with buffer at a $\mathrm{pH}$ of 4.0 (10 mM succinic acid $\mathrm{pH} 4.0,300 \mathrm{mM} \mathrm{KCl})$ or $\mathrm{pH} 7.5$ (10 mM HEPES pH 7.5, $300 \mathrm{mM} \mathrm{KCl}$ ). Images were taken with a Picoplus 5500 (Molecular Imaging) in the acoustic AC mode at room temperature. Cantilevers used were BL-AC40TS (tip radius: $<10 \mathrm{~nm}$, spring constant: $\sim 0.1 \mathrm{~N} / \mathrm{m}$, OLYMPUS). All images were recorded in buffer solution in liquid cells. To evaluate the longitudinal length of the channel, the height profile, cross-sectioning the highest peak of the channel as well as the mica surface in its course, was used.

Image processing. The averaged images of KcsA molecules were processed using a custom-made program running on Igor Pro 6.2. A reference molecule was picked up as a region of interest (ROI) and the molecules with similar features were collected using correlation pattern matching over the entire image. KcsA molecules with a normalized correlation coefficient over 0.9 to the reference image were averaged in four-fold symmetry.

MD simulation. The MD simulations were performed with using the AMBER9 package ${ }^{41}$. In order to minimize the dependency of the trajectories on the initial coordinates, ten different initial coordinates were generated for both the closed and open structures at the NVT ensemble $(310 \mathrm{~K})$. A periodic boundary condition was imposed. Electrostatic interactions were calculated using the particle mesh Ewald method ${ }^{42}$ with a $0.8 \mathrm{~nm}$ cutoff in real space. The cutoff of the Lennard-Jones interaction was $0.8 \mathrm{~nm}$. The bonds including those of $\mathrm{H}$ atoms, were constrained with the SHAKE algorithm ${ }^{43}$, enabling a time step of $2 \mathrm{fs}$.

The system was prepared in the following manner. First, the dioleoylphosphatidylcholine (DOPC) bilayer was equilibrated with the surrounding $\mathrm{KCl}$ solution for $10 \mathrm{~ns}$. The empirical potentials were used for water (TIP3P $)^{44}$, DOPC ${ }^{45}$, and ions ${ }^{46}$. The area per lipid after the equilibrium was confirmed to be the same as that reported. To avoid the aggregation of $\mathrm{KCl}$ clusters ${ }^{47}$, parameters for ion developed by Dang et al. were used. The MD simulation was performed at the NPT ensemble (1 bar, $310 \mathrm{~K}$ ) using Berendsen's barostat ${ }^{48}$. A vacant space was then created by removal of the DOPC and solution for filling the KcsA channel (PDB code: 1K4C) with F125 added to the C-terminal end (residues 22-125). The AMBER94 parameters were used for the $\mathrm{KcsA}_{\text {channel }}{ }^{49}$. E71 was protonated as in the previous study ${ }^{50}$. H25, E118, E120, and H124 were also protonated with the structure in the open state under an acidic condition $(\mathrm{pH}=4.0)$. Equilibrium simulation was performed for $10 \mathrm{~ns}$ at NPT ensemble ( 1 bar, $310 \mathrm{~K}$ ), where the $\mathrm{C}_{\alpha}$ in residues $22-124$ were under the influence of a weak harmonic constraint $\left(0.25 \mathrm{kcal} / \mathrm{mol} / \AA^{2}\right)$. The simulation for equilibration in the NVT ensemble $(310 \mathrm{~K}$ ) was continued for $10 \mathrm{~ns}$ after the constraint on the KcsA channel was removed.

The coordinates of the $\mathrm{C}_{\alpha}$ in residues 30-114 were constrained to those of a $1.7 \mathrm{~nm}$-wide open structure (PDB code: $3 \mathrm{~F} 7 \mathrm{Y}$ ) using a weak harmonic constraint $\left(0.25 \mathrm{kcal} / \mathrm{mol} / \mathrm{A}^{2}\right)$ for $50 \mathrm{~ns}$. Next, the coordinates of the same $\mathrm{C}_{\alpha}$ were induced to those of a $2.3 \mathrm{~nm}$-wide open structure (PDB code: $3 \mathrm{~F} 7 \mathrm{~V}$ ) with the same harmonic constraint for $50 \mathrm{~ns}$. Finally, the coordinates of the same $\mathrm{C}_{\alpha}$ were constrained to those of $3.2 \mathrm{~nm}$-wide open structure (PDB code: $3 \mathrm{~F} 5 \mathrm{~W}$ ) in the same way. Although the restraints were imposed on the $\mathrm{C}_{\alpha}$ atoms, the resultant structure was similar to that of $3 \mathrm{~F} 7 \mathrm{~V}$ in respect of the average $\mathrm{C}_{\alpha}-\mathrm{C}_{\alpha}$ distances at T112 of the diagonal subunits $(2.4 \mathrm{~nm})$.

For ten different initial coordinates, each coordinate was calculated for $20 \mathrm{~ns}$ under NVT ensemble without restraint and was used for the analyses. The total simulation time was $200 \mathrm{~ns}$ for both closed and open states. In the closed state, the system comprised of one KcsA channel, 119 DOPC, $30 \mathrm{~K}^{+}, 58 \mathrm{Cl}^{-}$, and 10704 water molecules. The system measured $77.3 \times 80.5 \times 87.1 \AA^{3}$.

Single-channel current recordings. Planar lipid bilayer method was used for the single-channel current measurements as reported previously ${ }^{38}$. A Teflon sheet of $0.5 \mathrm{~mm}$ thickness with a hole $(<100 \mu \mathrm{m}$ diameter $)$ was used as a partition which divided the two (cis and trans) compartments of a chamber. The planar lipid bilayer was formed on a hole by painting $20 \mathrm{mg} / \mathrm{mL} n$-decane (Nacalai, Kyoto, Japan) solution of 1-palmitoyl-2-oleoyl-sn-glycero-3-phospho-(1'-rac-glycerol) (Avanti Polar Lipids, Alabaster, AL). Electrolyte solution in the cis and trans chambers contained $200 \mathrm{mM} \mathrm{KCl}$ buffered by $10 \mathrm{mM}$ HEPES (pH 7.5) and $10 \mathrm{mM}$ succinic acid ( $\mathrm{pH} 4.0)$, respectively. The reference electrode was placed in the cis compartment, to which KcsA channels reconstituted in proteoliposomes were added. The current was recorded through an Axopatch 200B amplifier and Digidata 1322A digitizer (Molecular Devices) and the data were analyzed by using pCLAMP software (Molecular Devices, Sunnyvale, CA). The low pass filter was set to $2 \mathrm{kHz}$ for the cut-off frequency, and the data were sampled at $5 \mathrm{kHz}$.

1. Hille, B. Ion channels of excitable membranes. Sinauer Associates Inc: Sunderland, (2001).

2. Miller, C. An overview of the potassium channel family. Genome biol $\mathbf{1}$, REVIEWS0004 (2000).

3. A Doyle, D. et al. The structure of the potassium channel: molecular basis of $\mathrm{K}^{+}$ conduction and selectivity. Science 280, 69-77 (1998).

4. Jiang, Y. et al. Crystal structure and mechanism of a calcium-gated potassium channel. Nature 417, 515-22 (2002).

5. Kuo, A. et al. Crystal Structure of the potassium channel KirBac1.1 in the Closed State. Science 300, 1922-1926 (2003).

6. Jiang, Y. et al. X-ray structure of a voltage-dependent $\mathrm{K}^{+}$channel. Nature $\mathbf{4 2 3}$, 33-41 (2003).

7. Gulbis, J. M. \& Doyle, D. A. Potassium channel structures: do they conform? Curr. Opin. Struct. Biol. 14, 440-6 (2004).

8. Oiki, S. Gating Dynamics of the Potassium Channel Pore. Egelman, E. Ed. Comprehensive Biophysics, Vol 6, Channel Proteins, 31-67, Oxford, Academic Press, (2012).

9. Zhou, Y., Morais-Cabral, J., Kaufman, A. \& MacKinnon, R. Chemistry of ion coordination and hydration revealed by a $\mathrm{K}^{+}$channel-Fab complex at $2.0 \AA$ resolution. Nature 414, 43-48 (2001).

10. Morais-Cabral, J. H., Zhou, Y. \& MacKinnon, R. Energetic optimization of ion conduction rate by the $\mathrm{K}^{+}$selectivity filter. Nature 414, 37-42 (2001).

11. Roux, B. \& MacKinnon, R. The cavity and pore helices in the KcsA $\mathrm{K}^{+}$channel: electrostatic stabilization of monovalent cations. Science 285, 100-2 (1999).

12. Miloshevsky, G. V. \& Jordan, P. C. Permeation in ion channels: the interplay of structure and theory. Trends Neurosci. 27, 308-14 (2004).

13. Nimigean, C. M. \& Miller, C. $\mathrm{Na}^{+}$Block and Permeation in a $\mathrm{K}^{+}$Channel of Known Structure. J. Gen. Physiol. 120, 323-335 (2002).

14. Iwamoto, M. \& Oiki, S. Counting Ion and Water Molecules in a Streaming File through the Open-Filter Structure of the K Channel. J. Neurosci. 31, 12180-8 (2011).

15. Cuello, L. G., Jogini, V., Cortes, D. M. \& Perozo, E. Structural mechanism of C-type inactivation in $\mathrm{K}^{+}$channels. Nature 466, 203-8 (2010).

16. Long, S. B., Campbell, E. B. \& Mackinnon, R. Crystal structure of a mammalian voltage-dependent Shaker family K ${ }^{+}$channel. Science 309, 897-903 (2005).

17. Ye, S., Li, Y. \& Jiang, Y. Novel insights into $\mathrm{K}^{+}$selectivity from high-resolution structures of an open $\mathrm{K}^{+}$channel pore. Nat. Struct. Mol. Biol. 17, 1019-23 (2010).

18. Uysal, S. et al. Crystal structure of full-length KcsA in its closed conformation. Proc. Natl. Acad. Sci. USA 106, 6644-6649 (2009).

19. Uysal, S. et al. Mechanism of activation gating in the full-length KcsA K ${ }^{+}$channel. Proc. Natl. Acad. Sci. USA 108, 11896-9 (2011). 
20. Van Huizen, R., Czajkowsky, D. M., Shi, D., Shao, Z. \& Li, M. Images of oligomeric Kv beta 2, a modulatory subunit of potassium channels. FEBS lett. 457, 107-11 (1999).

21. Engel, A. \& Müller, D. Observing single biomolecules at work with the atomic force microscope. Nat. Struct. Biol. 7, 715-718 (2000).

22. Milhiet, P.-E. et al. High-resolution AFM of membrane proteins directly incorporated at high density in planar lipid bilayer. Biophys. J. 91, 3268-75 (2006).

23. Scheuring, S., Lévy, D. \& Rigaud, J.-L. Watching the components of photosynthetic bacterial membranes and their in situ organisation by atomic force microscopy. Bioch. Biophys. Acta 1712, 109-27 (2005).

24. Jarosławski, S., Zadek, B., Ashcroft, F., Vénien-Bryan, C. \& Scheuring, S. Direct visualization of KirBac3.1 potassium channel gating by atomic force microscopy. J. Mol. Biol. 374, 500-5 (2007).

25. Shinozaki, Y. et al. Direct Observation of ATP-Induced Conformational Changes in Single P2×4 Receptors. PLoS biol. 7, e103 (2009).

26. Mari, S. et al. Gating of the MlotiK1 potassium channel involves large rearrangements of the cyclic nucleotide-binding domains. Proc. Natl. Acad. Sci. USA 108, 20802-20807 (2011).

27. Shimizu, H. et al. Global twisting motion of single molecular KcsA potassium channel upon gating. Cell 132, 67-78 (2008).

28. Oiki, S., Shimizu, H., Iwamoto, M. \& Konno, T. Single-Molecular Gating Dynamics for the KcsA Potassium Channel. Adv. Chem. Phys. 146, 147-193 (2012).

29. Cuello, L. G., Cortes, D. M., Jogini, V., Sompornpisut, A. \& Perozo, E. A molecular mechanism for proton-dependent gating in KcsA. FEBS lett. 584, 1126-32 (2010).

30. Heginbotham, L., LeMasurier, M., Kolmakova-Partensky, L. \& Miller, C. Single streptomyces lividans $\mathrm{K}^{+}$channels: functional asymmetries and sidedness of proton activation. J. Gen. Physiol. 114, 551-60 (1999).

31. Thompson, A. N., Posson, D. J., Parsa, P. V. \& Nimigean, C. M. Molecular mechanism of $\mathrm{pH}$ sensing in KcsA potassium channels. Proc. Natl. Acad. Sci. USA 105, 6900-5 (2008).

32. Cortes, D. M., Cuello, L. G. \& Perozo, E. Molecular architecture of full-length KcsA: role of cytoplasmic domains in ion permeation and activation gating. J. Gen. Physiol. 117, 165-80 (2001).

33. Yuan, Z., Zhao, J. \& Wang, Z.-X. Flexibility analysis of enzyme active sites by crystallographic temperature factors. Protein Eng. 16, 109-114 (2003).

34. Cordero-Morales, J. F., Cuello, L. G. \& Perozo, E. Voltage-dependent gating at the KcsA selectivity filter. Nat. Struct. Mol. Biol. 13, 319-22 (2006).

35. Heinemann, S. H. \& Sigworth, F. J. Open channel noise. VI. Analysis of amplitude histograms to determine rapid kinetic parameters. Biophys. J. 60, 577-87 (1991).

36. Läuger, P., Stephan, W. \& Frehland, E. Fluctuations of barrier structure in ionic channels. Biochim. Biophys. Acta 602, 167-80 (1980).

37. Yellen, G. Ionic permeation and blockade in $\mathrm{Ca}^{2+}$-activated $\mathrm{K}^{+}$channels of bovine chromaffin cells. J. Gen. Physiol. 84, 157-86 (1984).

38. Iwamoto, M. et al. Surface structure and its dynamic rearrangements of the KcsA potassium channel upon gating and tetrabutylammonium blocking. J. Biol.Chem. 281, 28379-86 (2006).

39. Seantier, B. \& Kasemo, B. Influence of mono- and divalent ions on the formation of supported phospholipid bilayers via vesicle adsorption. Langmuir 25, 5767-72 (2009).

40. Sumino, A. et al. Reconstitution and AFM Observation of Photosynthetic Membrane Protein Assembly in Planar Lipid Bilayers. e-J. Surf. Sci. Nanotech. 9 , 15-20 (2011).

41. Case, D. A. et al. AMBER 9. 328 (University of California: San Francisco, 2006).

42. Essmann, U., Perera, L. \& Berkowitz, M. A smooth particle mesh Ewald method. J. Chem. Phys. 103, 31-34 (1995).
43. Ryckaert, J., Ciccotti, G. \& Berendsen, H. J. C. Numerical integration of the cartesian equations of motion of a system with constraints: molecular dynamics of n-alkanes. J. Comput. Phys. 23, 327-341 (1977).

44. Jorgensen, W., Chandrasekhar, J., Madura, J., Impey, R. \& Klein, M. Comparison of simple potential functions for simulating liquid water. J. Chem. Phys. 79, 926-935 (1983)

45. Siu, S., Vácha, R., Jungwirth, P. \& Böckmann, R. Biomolecular simulations of membranes: Physical properties from different force fields. J. Chem. Phys. 128, 125103 (2008).

46. Dang, L. Mechanism and thermodynamics of ion selectivity in aqueous solutions of 18-crown-6 ether: a molecular dynamics study. J. Am. Chem. Soc. 117, 6954-6960 (1995).

47. Auffinger, P., Cheatham III, T. E. \& Vaiana, A. C. Spontaneous formation of KCl aggregates in biomolecular simulations: A force field issue? J. Chem. Theo. Comput. 3, 1851-1859 (2007)

48. Berendsen, H. J. C., Postma, J. P. M., Van Gunsteren, W. F., DiNola, A. \& Haak, J. R. Molecular dynamics with coupling to an external bath. J. Chem. Phys. 81, 3684-3690 (1984).

49. Cornell, W. et al. A second generation force field for the simulation of proteins, nucleic acids, and organic molecules. J. Am. Chem. Soc. 117, 5179-5197 (1995).

50. Bernèche, S. \& Roux, B. Molecular dynamics of the KcsA $\mathrm{K}^{+}$channel in a bilayer membrane. Biophys. J. 78, 2900-17 (2000).

\section{Acknowledgment}

We thank Dr. Takayuki Uchihashi (Kanazawa Univ., Japan) for writing averaging program. We thank Prof. Hiroshi Onishi and Mr. Tomoki Sugihara (Kobe Univ., Japan) for helpful discussions. T.D. thanks PRESTO (JST) and Grant-in-Aid for Scientific Research on Innovative Areas 2006 (Molecular Science of Fluctuations toward Biological Functions)" (23107713) for funding. M.I. and S.O. thank Grant-in-Aid for Scientific Research (B) $(11020017)$ and $(23370067,21107508,21657038,20247016)$ from JSPS. A.S. thanks Grant-in-Aid for JSPS Fellows (23-02697) for funding. The simulations were performed using Research Center for Computational Science, Okazaki, Japan, and we thank Prof. Shinji Saito. This work was supported by the Joint Studies Program (2011-2012) of the Institute for Molecular Science. We thank Pacific Edit for reviewing the manuscript prior to submission.

\section{Author contributions}

A.S., T.S., M.I., T.D. and S.O. wrote the manuscript. A.S., T.S., M.I., T.D. and S.O. designed research. A.S., T.S. and M.I. performed experiments. A.S. T.S. and S.O. analyzed data. All authors reviewed the manuscript.

\section{Additional information}

Supplementary information accompanies this paper at http://www.nature.com/ scientificreports

Competing financial interests: The authors declare no competing financial interests. License: This work is licensed under a Creative Commons Attribution-NonCommercial-NoDerivs 3.0 Unported License. To view a copy of this license, visit http://creativecommons.org/licenses/by-nc-nd/3.0/

How to cite this article: Sumino, A., Sumikama, T., Iwamoto, M., Dewa, T. \& Oiki, S. The Open Gate Structure of the Membrane-Embedded KcsA Potassium Channel Viewed From the Cytoplasmic Side. Sci. Rep. 3, 1063; DOI:10.1038/srep01063 (2013) 